\title{
The Complex Process of the Health Technology Incorporation in Brazil
}

\author{
Áurea J. Chaves
}

$\mathrm{T}$ he transcatheter aortic valve implantation (TAVI, or TAVR, transcatheter aortic valve replacement), the treatment of choice for patients with severe aortic stricture deemed inoperable (class I, level of evidence B) and an alternative strategy for surgical valve replacement surgery in high-risk individuals (class Ila, level of evidence B), has been employed, to date, in over 700 patients in Brazil. This procedure was approved by the Brazilian Federal Council of Medicine in January 2012, after its evaluation as a non-experimental practice. This phase was subsequent to the approval of the device used in the procedure by the Brazilian Health Surveillance Agency (Agência Nacional de Vigilância Sanitária - ANVISA).

In the Brazilian system of health technology incorporation, compliance with these first two steps does not guarantee reimbursement by the private health care system or by the Brazilian Unified Health System (Sistema Único de Saúde - SUS). To ensure mandatory reimbursement by health plans, the procedure should integrate the List of Health Procedures and Events developed by the Brazilian National Regulatory Agency for Private Health Insurance and Plans (Agência Nacional de Saúde Suplementar - ANS), which is renewed every two years. To obtain reimbursement by SUS, an approval from the National Committee of Technology Incorporation (Comissão Nacional de Incorporação de Tecnologias - CONITEC) is required. CONITEC must issue a ruling within 180 days of request of the incorporation.

In the case of TAVI, in three opportunities the ANS did not include the technique in the List of Procedures. In the last update, which will be enforced from 2014, a Permanent Technical Group was created, which may deliberate in favor of the inclusion of new procedures before the regulatory two years. At CONITEC, Brazilian Society of Hemodynamics and Interventional Cardiology (Sociedade Brasileira de Hemodinâmica e Cardiologia Intervencionista $\mathrm{SBHCl}$ ) claimed for the inclusion of TAVI in March 2013, and recently the committee launched a public consultation for the incorporation of TAVI with the recommendation, in a low degree, to not incorporate this procedure. However, this recommendation can be modified after a public consultation, or can be opened to contestation, on the appeal level, to Ministry of Health.

Some time ago, ANS added, among the criteria for inclusion in the list, the economic evaluation of the procedure. Under SUS, despite the constitutional principle implying that healthcare is a right of all and a duty of the State, the access to healthcare is conditioned to the existence of budget resources. In order to claim again for the inclusion of TAVI in the private healthcare system, Queiroga et al., on behalf of $\mathrm{SBHCl}$, conducted a study of cost-effectiveness in order to include this intervention in the list of procedures. These authors developed a predictive model to evaluate the cost-effectiveness of the procedure in long-term. They demonstrated that the incorporation of TAVI by ANS would entail an incremental budgetary impact over the next five years that is consistent with other previously incorporated technologies. Siqueira and Abizaid, from Instituto Dante Pazzanese de São Paulo, in São Paulo, SP, Brazil, and Kodali and Leon, from Columbia University Medical Center/New York Presbyterian Hospital and Cardiovascular Research Foundation, New York, United States, in a correspondent editorial, estimated that over 45,000 patients could benefit from this intervention in Brazil - a fact that is hampered by the lack of reimbursement by the private healthcare system and SUS. These authors present evidence of the clinical value of TAVI, especially in prohibitive surgical risk patients: increased life expectancy and quality of life; maintenance of the procedure results after five years; and decrease of complications associated with the intervention with new generation devices. They complemented the analysis by revealing results of costeffectiveness studies of countries such as the United States, Canada, and England that, along with data published on this issue, exhibit costs that are similar to other medical treatments already incorporated by these institutions.

In another prominent article, Andrade et al., from Santa Casa de Marília, Marília, SP, Brazil, reported the outcomes of patients with acute myocardial infarction (AMI) undergoing primary percutaneous coronary intervention $(\mathrm{PCl})$, comparing those with and without resolution of the ST-segment. These authors identified the frequency, patient profile, and clinical consequences 
of those who developed this phenomenon. In a related editorial, Abelin and Quadros, from Instituto de Cardiologia, Fundação Universitária de Cardiologia, Porto Alegre (RS), explained that the analysis of ST-segment resolution after reperfusion therapy is a useful, simple, and inexpensive tool to assess microvascular reperfusion that helps to reclassify the patient risk, especially in those with Thrombolysis in Myocardial Infarction (TIMI) 3 flow after primary $\mathrm{PCl}$. These authors review the methods used and their role as a prognostic indicator and as a research tool in contemporary times.

Complementing this edition are other important articles, addressing the predictors of thrombo aspiration failure in primary $\mathrm{PCl}$; influence of location of lesions in saphenous vein grafts in clinical outcomes after PCl; results of $\mathrm{PCI}$ by radial route in patients with stable and unstable coronary artery disease; outcomes of diabetic patients treated with drug-eluting stents (DES) from the Safira Registry; changes in the population profile and results of $\mathrm{PCl}$ in the Angiocardio Registry; evolution of patients with cardiogenic shock due to AMI with ST-segment elevation; aortic valvuloplasty in patients with clinical condition in extremis; interference of reprocessed sheaths in occlusion of the radial artery after cardiac catheterization; and usefulness of coronary angiography and nuclear magnetic resonance imaging in the diagnosis of ischaemic cardiomyopathy.

Enjoy your reading!

Áurea J. Chaves

Editor 